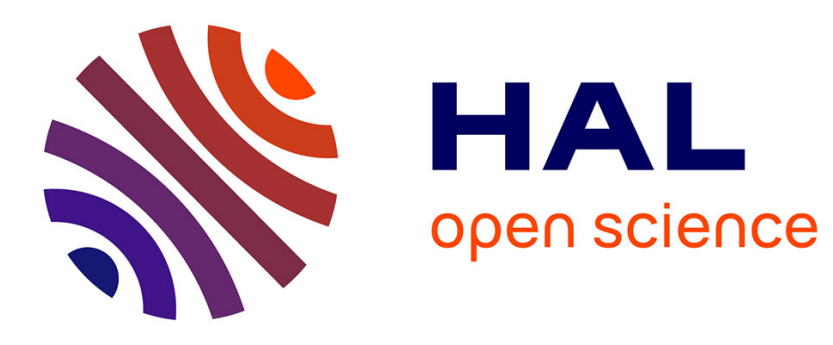

\title{
Synthesis of Uniform, Monodisperse, Sophorolipid Twisted Ribbons
}

Anne-Sophie Cuvier, Florence Babonneau, Jan Berton, Christian V. Stevens, Giulia C. Fadda, Isabelle Genois, Patrick Le Griel, Gerard Pehau-Arnaudet, Niki Baccile

\section{To cite this version:}

Anne-Sophie Cuvier, Florence Babonneau, Jan Berton, Christian V. Stevens, Giulia C. Fadda, et al.. Synthesis of Uniform, Monodisperse, Sophorolipid Twisted Ribbons. Chemistry - An Asian Journal, 2015, 10 (11), pp.2419-2426. 10.1002/asia.201500693 . hal-01275042

\section{HAL Id: hal-01275042 \\ https://hal.science/hal-01275042}

Submitted on 14 Feb 2017

HAL is a multi-disciplinary open access archive for the deposit and dissemination of scientific research documents, whether they are published or not. The documents may come from teaching and research institutions in France or abroad, or from public or private research centers.
L'archive ouverte pluridisciplinaire HAL, est destinée au dépôt et à la diffusion de documents scientifiques de niveau recherche, publiés ou non, émanant des établissements d'enseignement et de recherche français ou étrangers, des laboratoires publics ou privés. 


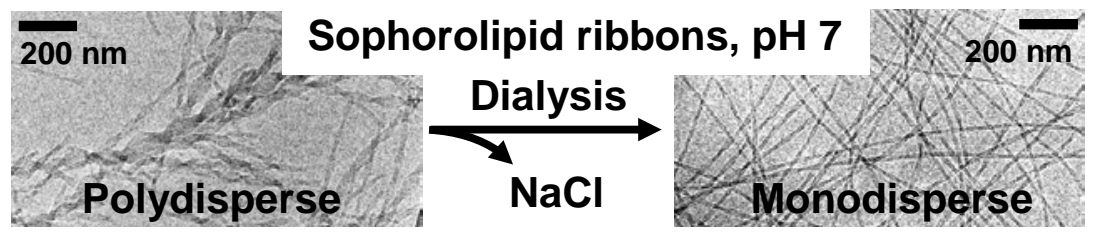

The acidic form of saturated sophorolipids can form in water, at $\mathrm{pH} 7$, self-assembled twisted ribbons, the cross-sectional monodispersity of which $(13.0 \pm 1.5 \mathrm{~nm})$ can easily be controlled by removing $\mathrm{NaCl}$ from the parent colloidal suspension using a simple dialysis step, as shown by a combination of SANS and cryo-TEM.

Keywords: acidic sophorolipids, saturated sophorolipids, twisted ribbons, modisperse colloidal suspension, cryo-TEM, SANS, self-assembly 


\title{
Synthesis of uniform, monodisperse, sophorolipid twisted ribbons
}

\author{
Anne-Sophie Cuvier, ${ }^{1}$ Florence Babonneau, ${ }^{1}$ Jan Berton, ${ }^{2}$ Christian V. Stevens, ${ }^{2}$ Giulia Fadda, ${ }^{3}$ Isabelle \\ Genois, ${ }^{1}$ Patrick Le Griel, ${ }^{1}$ Gérard Péhau-Arnaudet, ${ }^{4}$ Niki Baccile ${ }^{1 *}$
}

[1] Sorbonne Universités, UPMC Univ Paris 06, CNRS, Collège de France, UMR 7574, Chimie de la Matière Condensée de Paris, F-75005 Paris, France. Corresponding author : niki.baccile@upmc.fr [2] SynBioC Research Group, Departement of Sustainable Organic Chemistry and Technology, Faculty of Bioscience Engineering, Ghent University, Coupure links 653, B-9000 Gent, Belgium [3] Laboratoire Léon Brillouin, LLB, CEA Saclay, F-91191 Gif-sur-Yvette Cedex, France [4] Institut Pasteur, UMR3528, 28 Rue du Docteur Roux, F-75015, Paris, France

\begin{abstract}
Control over size monodispersity in chiral self-assembled systems is important for potential applications like templating, tissue engineering or enantioselective chromatography, just to cite some. In this context, it was reported that the saturated form of sophorolipids (SL), a bioderived glycolipid, are able to form self-assembled twisted ribbons in water at neutral $\mathrm{pH}$. Here, we show the possibility to control their size dispersion, generally comprised between $10 \mathrm{~nm}$ and $40 \mathrm{~nm}$ after synthesis to a value of $13.5 \pm 1.5 \mathrm{~nm}$, by a simple dialysis step eliminating the excess of $\mathrm{NaCl}$. We use Transmission Electron Microscopy under cryogenic conditions (cryo-TEM) combined with Small Angle Neutron Scattering (SANS) to characterize the ribbon dispersion both visually and statistically. Two negative controls show the importance of salt in the aggregation process of the ribbons.
\end{abstract}

\section{INTRODUCTION}

Self-assembly at a supramolecular scale is a fascinating phenomenon that is studied by various scientific communities, including life and chemical science. In particular, giant self-assembled aggregates like fibers, nanotubes and ribbons are highly attractive for many reasons, going from understand- 
ing a number of diverse phenomena occurring in living systems to their use in material science. Examples are the functional mechanisms of actin, ${ }^{1}$ an important filamentous protein (F-actin) present in most eukaryotic cells and involved in cell mobility, or amyloid, ${ }^{2}$ a fibrillar protein whose self-assembly is involved in the Alzheimer disease, to the use of collagen, ${ }^{3}$ another ubiquitous fibrillar protein present in living tissues, or amphiphilic peptides ${ }^{4}$ in the development of materials for biomedical applications like tissue engineering, ${ }^{5,6}$ or spinal cord repair. ${ }^{7}$ On another level, chiral fibers of perfectly controlled shape have been employed as templates for silica ${ }^{8}$ and carbon $^{9}$ materials, both being interesting candidates as solid supports for enantioselective chromatography. ${ }^{10}$ Hollow lipidic infinitely long nanotubes of controlled diameter have been used for applications in the field of attoliter solution chemistry ${ }^{11}$ while cellulose nanorods of controlled size dispersion have been used to develop photonic and other functional materials ${ }^{12}$. Moreover, they were also applied to better understand the origin of color in Pollia condensata, a fruit in which the color is caused by Bragg reflection of helicoidally stacked cellulose microfibrils, forming multilayers in the cell walls of the epicarps. ${ }^{13}$

In a recent communication, we have shown how bioderived bolaform glycolipids are able to self-assemble into twisted ribbons, ${ }^{14}$ an interesting morphology generally reported as being a precursor for lipid nanotube formation and described for various molecular systems including gemini surfactants, ${ }^{15}$ peptide amphiphiles ${ }^{16,17}$ or peptides. ${ }^{18}$ In particular, we employed a saturated form of acidic sophorolipids, molecules that are naturally produced by the yeast $S$. bombicola in large amounts. ${ }^{19}$ Known as biosurfactants and used as such in detergents, recent work has shown the variety of selfassembled aggregates capable to be formed spontaneously, or driven by setting the pH in water. ${ }^{14,20,21,22}$ Our work ${ }^{14}$ has shown for the first time that these compounds can form ribbons with nanoscale chirality. However, these structures had the disadvantage of being heterogeneous both in length and width, undergoing both aggregation and bundling, making them interesting objects but of little practical use due to the high polydispersity. The problem of controlling the morphology and polydispersity in size of fibrillar self-assembled objects is quite general and it was already commented before for asymmetric bolaform lipids. ${ }^{11}$ If Oda et al. ${ }^{15}$, as early as 1999 , have used thermodynamics arguments to predict, for instance, the impact of enantiomeric excess on the type of ribbon curvature (cylindrical vs. saddle like) and width polydispersity in gemini surfactants systems, the same authors have shown several years later ${ }^{23}$ the impact of impurities (e.g., presence of $\mathrm{Br}^{-}$), kinetics of formation, chemical composition (e.g., chain length of surfactant) on polydispersity. Finding a robust way to obtain good size monodispersity is still a hardto-control challenge and difficult to generalize. In the sophorolipid-based self-assembled systems, the problem of polydispersity has never been addressed so far, and it is of particular relevance because these 
compounds, as most bio-based molecules, are intrinsically characterized by a natural chemical variation in one single batch. For instance, sophorolipids are commonly obtained in a mixture of C16 and C18 aliphatic chains, ${ }^{24}$ with a majority (generally $>80 \mathrm{~mol} \%$ ) of the C18 compound, or mixtures of saturated, mono- and di-unsaturated species, mixtures of acetylated and non-acetylated, etc.... In the present work, we use fully saturated, non-acetylated, acidic sophorolipids, ${ }^{14}$ thus limiting broad chemical variations, but spurious presence of $\mathrm{C}_{18}-\mathrm{C}_{16}$ mixtures are, for instance, not excluded. For these reasons, the goal of this work is to show that it is possible to produce a homogeneous network of twisted ribbons, without losing the nanoscale chirality, by simply playing on the ionic strength of the solution and using the natural mixture of sophorolipids: the salt generated in the synthesis process is responsible for the aggregation phenomena and, upon its removal, a much better width monodispersity (13.5 $\pm 1.5 \mathrm{~nm})$ of the ribbons is easily obtained.

\section{Experimental}

Chemicals. All solutions (sophorolipids and acid/base solutions) were prepared with deionized water. $5 \mathrm{M} \mathrm{NaOH}$ solutions were obtained by the dissolution of $5 \mathrm{~g}$ of solid sodium hydroxide pellets (Sigma Aldrich) in $25 \mathrm{~mL}$ of deionized water. 0.5 and $0.05 \mathrm{M} \mathrm{HCl}$ solutions were prepared by diluting $37 \mathrm{w} \%$ hydrochloric acid (Sigma Aldrich) (1.23 mL and $0.123 \mathrm{~mL}$ respectively) in $25 \mathrm{~mL}$ of deionized water. Cellulose membranes for dialysis with a molecular-weight cutoff of 3.500 Da were purchased from Spectrum Laboratories, Inc. (USA). The crude sophorolipids extract (Sopholiance_S, Batch N 11103A) has been obtained by Soliance (Pomacle, France). This compound contains a mixture of acetylated, lactonic and acidic sophorolipids. This product was treated under alkaline conditions followed by acidification and pentanol extraction in order to recover only the non-acetylated acidic form of sophorolipids. The fully saturated version of this compound has been obtained with a classical catalytic hydrogenation step $\left(\mathrm{Pd} / \mathrm{C} / \mathrm{H}_{2}\right)$. The entire synthesis procedure including NMR and HPLC analyses are provided in Ref. 14: the same compounds have been used to perform the experiments in this work.

Sample preparation. Acidic sophorolipids (C18:0) with a concentration of $5 \mathrm{mg} / \mathrm{mL}$ were solubilized at $\mathrm{pH} 11$ with $5 \mu \mathrm{L}$ of a $5 \mathrm{M} \mathrm{NaOH}$ solution in deionized water. $\mathrm{pH}$ was further decreased to 7 with $\mu$ molar amounts of 0.5 and $0.05 \mathrm{M} \mathrm{HCl}$ solutions. $\mathrm{pH}$ measurements were done with a classical pH-meter. A freshly prepared solution of C18:0 sophorolipids was then dialyzed against deionized water in a cellulose membrane with a molecular-weight cutoff of 3.500. Water was changed and after 3 days all sodium chloride was removed from the sophorolipid solution. The removed salt concentration can be calculated by taking into account the number of moles of $\mathrm{NaOH}\left(2.5 \times 10^{-5} \mathrm{~mol}\right)$ and of $\mathrm{HCl}\left(5.0 \times 10^{-6} \mathrm{~mol}\right)$ added 
to the solution. The amount of $\mathrm{NaCl}$ in solution is then equal to $\mathrm{n}_{\mathrm{Cl}}{ }^{-}$, thus $\mathrm{n}_{\mathrm{NaCl}}$ is estimated to be $5.0 \mathrm{x}$ $10^{-6} \mathrm{~mol}$ and $\mathrm{NaCl}$ concentration $(\mathrm{V}=1 \mathrm{~mL})$ is then $5.0 \times 10^{-3} \mathrm{~mol} / \mathrm{L}$.

Cryo-Transmission Electron Microscopy (cryo-TEM).These experiments were carried out at the PFMU, Institut Pasteur (Paris, France) on a Tecnai F20 microscope operating at $200 \mathrm{KV}$ and equipped with a Gatan ultrascan 4000 camera. Image acquisition and processing was done with the DigitalMicrograph ${ }^{\mathrm{TM}}$ software. Cryofixation was done on a EMGP, Leica (Austria) instrument. A drop of sophorolipid solution was put on a holey carbon coated TEM copper grid (Quantifoil R2/2, Germany) and after removal of excess solution on the grid, it was plunged into liquid ethane and then stored at $-180^{\circ} \mathrm{C}$ in liquid nitrogen until observation on the microscope.

Unidirectional metal-shadowing using TEM. For this experiment we used a FEI Tecnai 120 Twin microscope operating at $120 \mathrm{kV}$ equipped with a Gatan Orius SC1000 CCD numeric camera 4k x 4k. The specimens were sputtered on freshly cleaved mica and let dry prior to platinum and carbon evaporation. The unidirectional shadowing of the specimens was performed in a Quorum Q150R ES device under high vacuum and at room temperature. $1.4 \mathrm{~nm}$ platinum was deposited at an elevation angle of $45^{\circ}$. An additional carbon layer of $6 \mathrm{~nm}$ was deposited perpendicularly on top of the metal films to stabilize and to reinforce the replicas. The replicas were then floated on water and collected on TEM grids for observation in the classical TEM mode

Small Angle Neutron Scattering (SANS). These experiments were performed on the PACE beamline at the Léon Brillouin Laboratory (LLB, Orphée Reactor, Gif-sur-Yvette, France). Three spectrometer configurations were chosen to cover different q-ranges: the high angle region $2.9 \cdot 10^{-2} \AA^{-1}<\mathrm{q}<3.0 \cdot 10^{-1}$ $\AA^{-1}$ with a neutron wavelength, $\lambda$, of $6 \AA$ and a sample-to-detector distance, D, of $1 \mathrm{~m}$; a medium angle region which covers a q-range $6.9 \cdot 10^{-3} \AA^{-1}<\mathrm{q}<7.3 \cdot 10^{-2} \AA^{-1}$ at $\lambda=6 \AA$ with $\mathrm{D}=4.7$; a small angle 2.4 $\cdot 10^{-3} \AA^{-1}<\mathrm{q}<2.6 \cdot 10^{-2} \AA^{-1}$ with a neutron wavelength, $\lambda$, of $17 \AA$ and a sample-to-detector distance, $\mathrm{D}$, of $4.7 \mathrm{~m} . \mathrm{q}$ is defined as $(4 \pi / \lambda) \sin \theta / 2$, where $\theta$ is the scattering angle between the incident and the scattered neutron beams. All samples for SANS have been prepared in $\mathrm{D}_{2} \mathrm{O}$, including the preparation of a $5 \mathrm{M}$ NaOD solution ( $\mathrm{NaOH}$ is dissolved in $\mathrm{D}_{2} \mathrm{O}$ using the same amounts previously described) and a commercial (Aldrich) $\mathrm{DCl} 37 \%$ solution to prepare diluted acidic solution (in $\mathrm{D}_{2} \mathrm{O}$ ). Dialysis was performed against $\mathrm{D}_{2} \mathrm{O}$. Dialyzed sophorolipid solutions were measured in $2 \mathrm{~mm}$ quartz cells at $\mathrm{T}=$ $25^{\circ} \mathrm{C}$. Data treatment was done with the PAsiNET.MAT software package provided at the beamline which is available free of charge. The scattering intensity is obtained from the determination of the number of neutrons in the incident beam and the detector cell solid angle. Data were corrected for the ambient background, empty cell scattering, neutron beam transmission and detector efficiency and nor- 
malized to the neutron beam flux. ${ }^{25}$ Incoherent signal was substracted by measuring the background $\left(100 \% \mathrm{D}_{2} \mathrm{O}\right)$ value at high-q values $\left(\mathrm{q}>0.3 \AA^{-1}\right)$.

Circular Dichroism. Optical activity of chiral molecules has been measured on a Jasco J-810 spectropolarimeter between $200 \mathrm{~nm}$ and $300 \mathrm{~nm}$ with a $0.1 \mathrm{~nm}$ step. A previously dialyzed solution of C18:0 sophorolipids at $\mathrm{pH} 7$ and a concentration of $5 \mathrm{mg} / \mathrm{mL}$ was loaded in a $1 \mathrm{~mm}$ quartz cuvette and then placed in the spectropolarimeter for measurement.

Trapdor ${ }^{1} \mathrm{H}\left\{{ }^{23} \mathrm{Na}\right\}$ Magic Angle Spinning Solid State Nuclear Magnetic Resonance (MAS ssNMR). ${ }^{1} \mathrm{H}$ and ${ }^{23} \mathrm{Na}$ solid state NMR experiments have been run on a $16.4 \mathrm{~T}$ Bruker Avance III spectrometer. ${ }^{1} \mathrm{H}$ and ${ }^{23} \mathrm{Na}$ chemical shifts have been calibrated, respectively, on TMS and NaCl. The Magic Angle Spinning (MAS) rate was $20 \mathrm{KHz}$ using $2.5 \mathrm{~mm}$ zirconia rotor. The $90^{\circ}$ pulse for ${ }^{23} \mathrm{Na}$ was $3.5 \mu \mathrm{s}$ at $138 \mathrm{~W}$ while the ${ }^{1} \mathrm{H} 90^{\circ}$ pulse was set to $3.5 \mu$ s at $38 \mathrm{~W}$. The power on the ${ }^{23} \mathrm{Na}$ channel was set to $10^{-12} \mathrm{~W}$ and $138 \mathrm{~W}$ for, respectively, the non-irradiated and irradiated states. We used a TRAPDOR pulse sequence implemented in the TOPSPIN (version 3.2) software. In a typical TRAPDOR experiment, ${ }^{26,27}$ a Hahn echo is set on the ${ }^{1} \mathrm{H}$ channel while a continuous irradiation is set on the ${ }^{23} \mathrm{Na}$ channel during the spin echo delay. Two experiments are systematically run each at given echo delay, without and with irradiation on the ${ }^{23} \mathrm{Na}$ channel. If the ${ }^{1} \mathrm{H}$ and ${ }^{23} \mathrm{Na}$ nuclei are magnetically coupled (e.g., close in space), the ${ }^{1} \mathrm{H}$ signal will be out of phase after ${ }^{23} \mathrm{Na}$ irradiation and the intensity of the corresponding ${ }^{1} \mathrm{H}$ spectrum will be affected. For the positive control, we used $\mathrm{NaH}_{2} \mathrm{PO}_{4}$, while for the negative control, we used a physical mixture of $\mathrm{NaCl}$ and adamantane.

\section{RESULTS AND DISCUSSION}

The effect of dialysis on the dispersion of sophorolipid fibers can first be observed using cryoTEM microscopy shown in Figure 1, in which the images before and after dialysis are displayed. We also compare the latter to a dialyzed sample to which $\mathrm{NaCl}$ is added on purpose. Figure $1 \mathrm{~A}$ shows an overview of the network of SL fibers synthesized as such. This sample is characterized by fibers with an inhomogeneous length and width. In particular, broad spherical aggregates are systematically observed (refer to arrow) next to smaller pieces of fibers of uneven length. Upon dialysis (Figure 1B), the fibers are homogeneously dispersed in the solution and no aggregates are detected. Upon magnification, the system as-such (Figure 1D,G) illustrates the presence of various fibrillar aggregates of unequal width, as pointed out by the black arrows. As previously described, ${ }^{14}$ the average width is $33.5 \pm 6.5 \mathrm{~nm}$ but aggregation phenomena are strongly evident, thus making the real size distribution much wider. The fibers display nanoscale chirality, also thoroughly described in ref. 14; in that case, the pitch was reported to be $241 \pm 38 \mathrm{~nm}$ at $\mathrm{pH}=6$ and $5 \mathrm{mg} / \mathrm{mL}$, thus making a variation of about $16 \%$. After salt removal, the 
fibers keep their twisted chirality, as shown in Figure 1E,H with an enhanced homogeneity both in terms of width $(13 \pm 3 \mathrm{~nm})$ and chiral pitch $(188 \pm 11 \mathrm{~nm})$. In addition, no aggregation among individual fibers is observed. Considering their length, the aspect ratio for the fibers in the dialyzed system is practically infinite. The estimated amount of $\mathrm{NaCl}$ in the as-synthesized system is $5 \mathrm{mM}$ and if one introduces this quantity in a dialyzed sample solution, it is possible to observe the overall changes by cryo-TEM. Figure 1C illustrates this point; at the same scale length as presented before, it is possible to observe again massive spheroidal aggregates of fibers (see arrow) surrounded by individual ones polydisperse in size and length. At higher magnification (Figure 1F,I), one can observe again various types of twisted ribbons, either alone or in bundles (arrow in Figure 1CI).

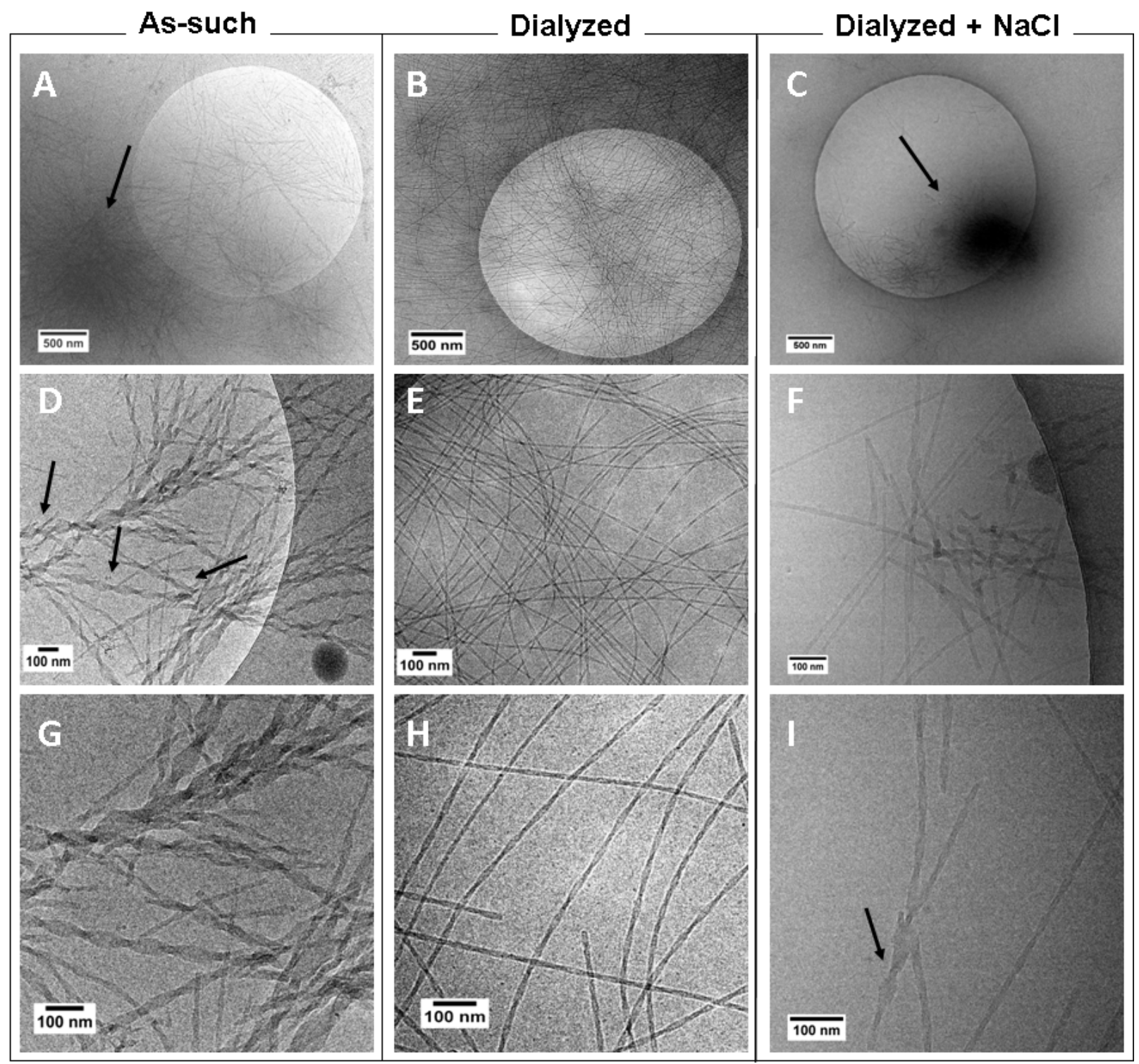

Figure 1 - Cryo-TEM images of self-assembled sophorolipid twisted ribbons. Images A, D, G correspond to assynthesized samples, which includes the sophorolipid twisted ribbons and $\mathrm{NaCl}$; images $\mathrm{B}, \mathrm{E}, \mathrm{H}$ correspond to a dia- 
lyzed, NaCl-free, solution of twisted ribbons and images C, F and I display dialyzed sophorolipid solution to which NaCl has been intentionally added.

The fact that chirality is preserved before and after dialysis is also demonstrated by the circular dichroism (CD) signal shown in Figure 2. We had previously shown that the same system at a pH exceeding 8 does not provide a signal in CD at all. ${ }^{14}$ Here, we show that such a signal is systematically detected below $250 \mathrm{~nm}$. Interestingly, by adding $\mathrm{NaCl}$ to a dialyzed sophorolipid solution one still observes the same signal in $\mathrm{CD}$ thus indicating that salt does not affect the formation of the twisted ribbon, as also suggested by cryo-TEM. It would be interesting to know whether the system is constituted by left- or right-handed ribbons and which is their respective proportion. Unfortunately, this quantification is not possible through $\mathrm{CD}$, as we lack a reference constituted by one of the two enantiomers alone. Cryo-TEM, although providing very clear images, is also not suitable to perform such a task because the lack of depth makes interpretation of the sense of chirality a harsh, non-reliable, task. To evaluate the sense of chirality and the relative population, we have made a Pt replica of the ribbons using a shadowing method (described in the experimental section) and analyzed the data under classical TEM acquisition conditions. Figure S1 shows the TEM images of a large number of dialyzed C18:0 sophorolipid chiral fibers. First of all, one can see that fiber chirality is preserved upon drying the sample, as indicated by the white arrows. To this regard, one can safely say that drying does not affect the fibers integrity. However, differently than what one can see in cryo-TEM, the nature of the ribbon curvature and aggregation state seem to be affected, because one can clearly see helical fibers (e.g., arrow in Figure S1b), having a cylindrical curvature, in addition to twisted fibers, which are the only objects that one can see in cryo-TEM. Strong aggregation upon drying (see, for instance, Figure S1c) is also documented, while this is hardly seen in the cryo-TEM experiments. These features are also observed in the Pt-replica of the 3-days aged system (images not shown). As far as the sense of chirality is concerned, one can observe both left- (Figure S1a-b) and right-handed (Figure S1c-d) twists on the same grid, with a slight majority of the latest. The following observations should induce strong precautions: 1) since a CD signal is always observed, one of the two forms must be predominant. However, at the moment, since we are not able to quantify CD data, we cannot affirm whether the CD signal is attributed to a single enantiomeric form or to a simple excess of sense of chirality with respect to the other one; 2) quantification could only be performed on about 50 individual fibers but this value should be cautiously interpreted because most of the grids are characterized by aggregated bundles, the exact number of which is difficult to evaluate; 3) replicas are obtained on dried samples and whether drying has an effect on the sense of chirality (through aggregation phenomena, for instance) is still an issue; as already commented, be- 
sides the fact that chiral fibers are preserved upon drying, we also observe strong aggregation phenomena, hardly observed in solution according to both cryo-TEM and SANS, as discussed later.

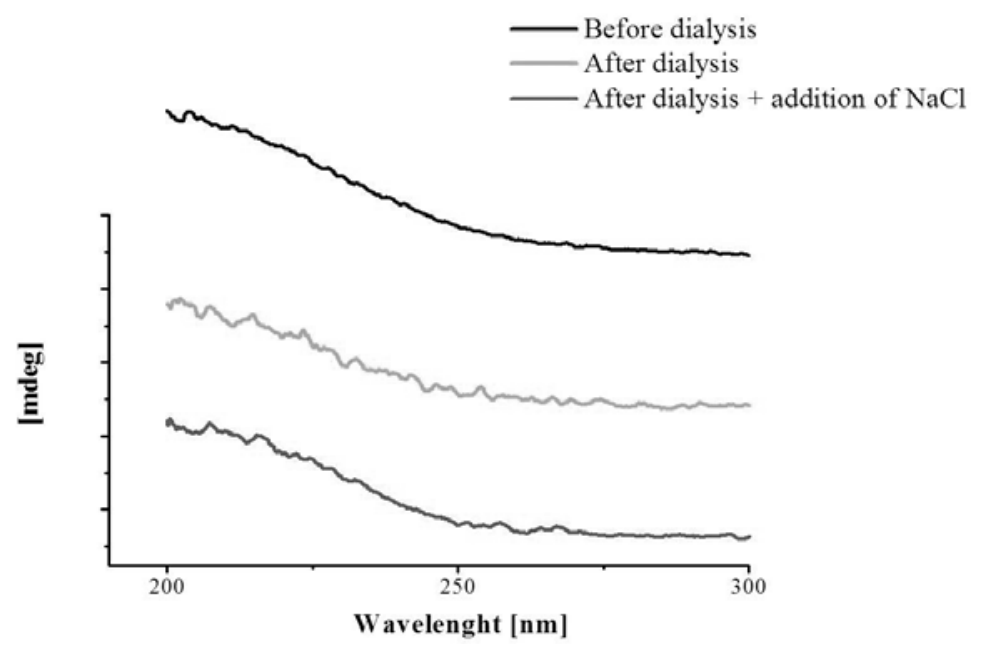

Figure 2 - Circular dichroism experiment performed on sophorolipid twisted ribbons before and after dialysis and on a dialyzed sample to which $\mathrm{NaCl}$ has been added. Data have been rescaled on the y-axis for readability purposes.

Since a typical dialysis procedure runs over at least three days, we verified that the monodispersion only depends on the salt concentration and not on aging time. In Figure 3 we compare the cryoTEM results of a typical ribbon-containing solution immediately after ribbon formation, and after three days of aging with and without dialysis. The starting solution shows the presence of micron-scale spheroidal aggregates (Figure 3a,b) composed of aggregated ribbons (Figure 3c) and surrounded by spherical particles. These objects are commonly found in fresh ribbon-containing solutions. After three days of aging at room temperature, the micron-sized aggregates are still observed but they show a quite clear interconnection formed by elongated ribbons which now cover the entire carbon support (Figure 3d): the fibers have increased in length. A closer look at them (Figure 3e,f) shows a network of chiral objects, similar in nature to what is found in the initial stage of their formation. At this stage, it seems that time has no effect on the type of curvature (twisted vs. cylindrical), as found in other systems. ${ }^{28}$ Figure 3e,f also shows fibers with large variations in terms of their cross-sections, ranging from $5 \mathrm{~nm}$ to more than $30 \mathrm{~nm}$, a result which is consistent and repeatable among the experiments (see for instance Figure $1 A, D, G)$. The analysis of the cross-section distribution, presented in the histogram in Figure 3 (black bars), shows two populations of ribbons centered at about $12 \mathrm{~nm}$ (80\%) and $35 \mathrm{~nm}$ (20\%). If the sample is dialyzed over the same time span, the fibrillar network is now more homogeneously dispersed over the entire grid (Figure 1B and Figure 3g), whereas the micronic aggregates are rather substituted by bundles of fibers that spontaneously form in water (Figure 3g). A closer look (Figure 3h,i) at the fiber 
dispersion shows a more homogenous population in terms of cross-sections and the quantification of their size distribution is given by the light gray bars in the histogram in Figure 3: the cross section of 95 $\%$ of the fibers falls between $12 \mathrm{~nm}$ and $15 \mathrm{~nm}$, thus providing an average value of $13.5 \pm 1.5 \mathrm{~nm}$. These experiments strongly support the idea that time contributes to fiber elongation, while $\mathrm{NaCl}$ controls fiber aggregation and polydispersity.
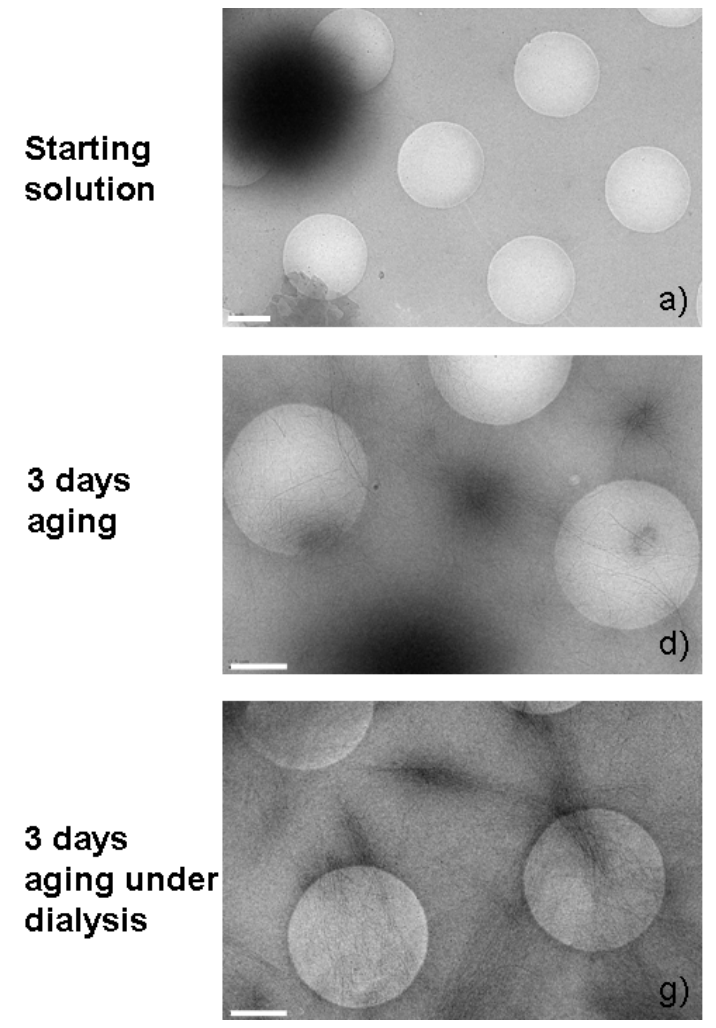
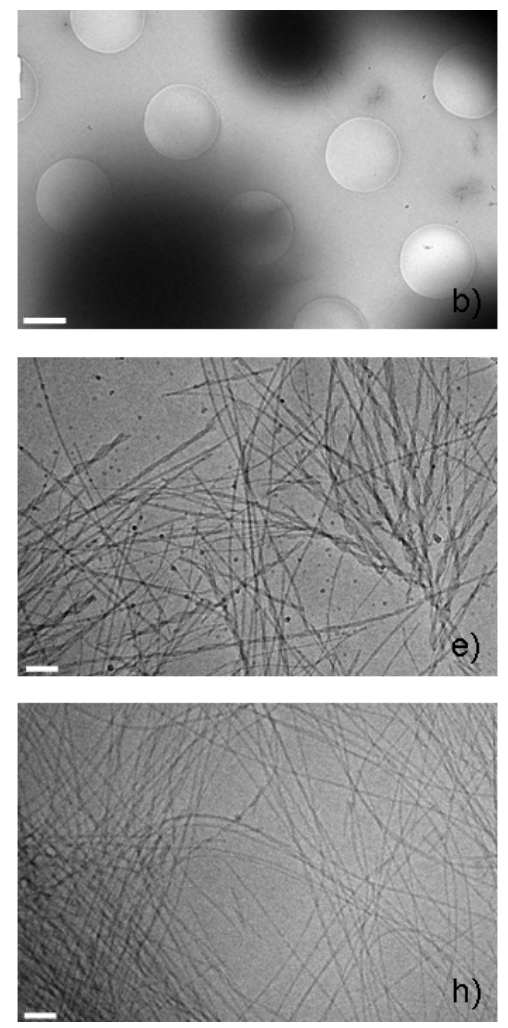
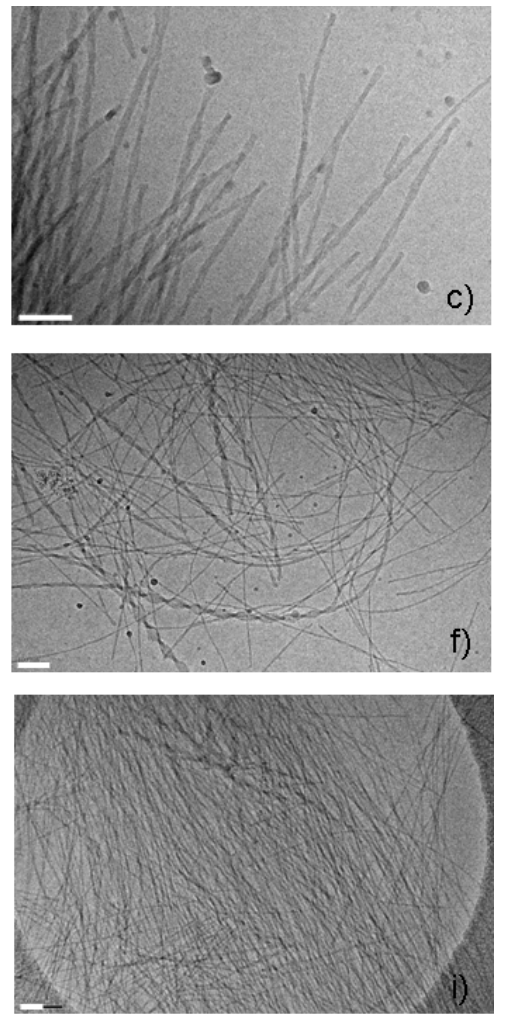

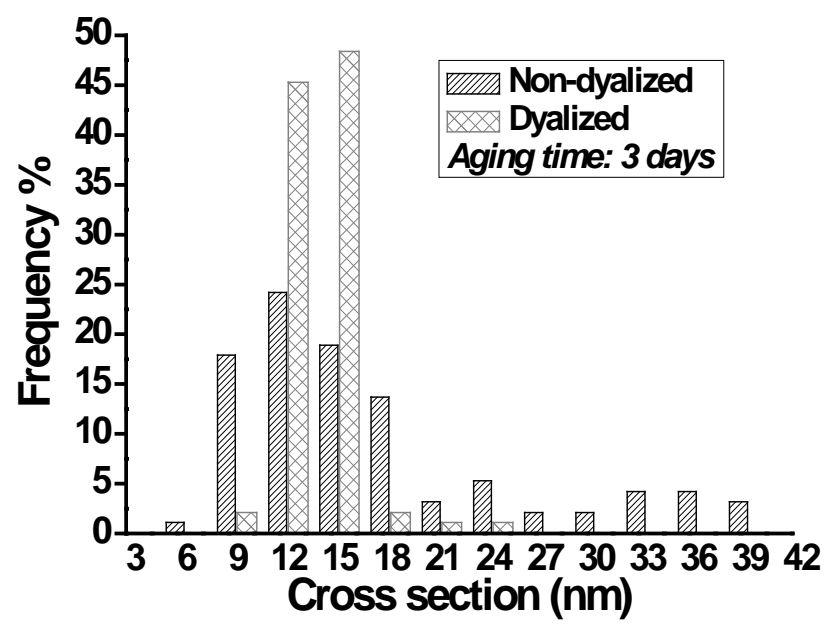

Figure 3 - a-f) Cryo-TEM images acquired at two aging times for the as-synthesized sample (immediate after synthesis and 3-days aging). g-i) Cryo-TEM images of the dialyzed sample after 3-days aging. Scale bars: images a,b,d,g= 1 $\mu \mathrm{m}$; images c,e,f,h,i= $100 \mathrm{~nm}$. The histogram reports the frequency \% (about 100 fibers counted) in terms of fibers cross section after 3 days aging both for the as-synthesized and dialyzed samples. 
Small Angle Neutron Scattering (SANS) is used hereafter to corroborate the results obtained by cryo-TEM. Chiral nanoscale fibers are characterized by a twisted flat monolayer of sophorolipid, as previously described. ${ }^{14}$ The typical SANS intensity is then expected to have a -2 dependency towards the wavevector $\mathrm{q}$ in the log-log scale. ${ }^{29}$ SANS experiments performed on the as-synthesized (stars) and dialyzed (full squares) systems are shown in Figure 4. In both cases, the slope at $\mathrm{q}<0.02 \AA^{-1}$ is very close but a difference exists between them; a $-1.83 \pm 0.06$ slope is measured in the as-synthesized system with respect to a $-2.11 \pm 0.12$ slope for the dialyzed material. Interestingly, when $\mathrm{NaCl}$ is added to the dialyzed system, the corresponding spectrum (hollow circles) is strictly superimposable with the assynthesized material, thus showing that the presence of salt directly affects the entire self-assembled fibers. It is still unclear what the origin of such a small difference in terms of slope could be. Integer values of the $\log (\mathrm{I}) \mathrm{vs} . \log (\mathrm{q})$ slopes are generally expected for systems the shape of which can be ideally described by a well-defined form factor expression. Typical values are $-1,-2$ and -4 for, respectively, a cylindrical, flat and spherical interface. In a polydisperse system, one generally measures the same slope dependency but the typical minima of the scattering function are averaged out over all sample sizes. In the present case, cryo-TEM images and CD show that the fibrillar twisted ribbon shape with a flat surface is not affected by the dialysis process; however, aggregation and polydispersity are minimized. Thus, the -1.83 slope can be considered as being the spectral signature of the fiber aggregation (polydispersity does not affect the slope), where the non-integer value is rather related to the fractal dimension of the aggregated flat ribbons. Non-integer values of $\log (\mathrm{I}) \mathrm{vs}$. $\log (\mathrm{q})$ are quite common for entangled networks, gels, aggregated particles with respect to the corresponding monodispersed, nonaggregated systems. ${ }^{30}$ 


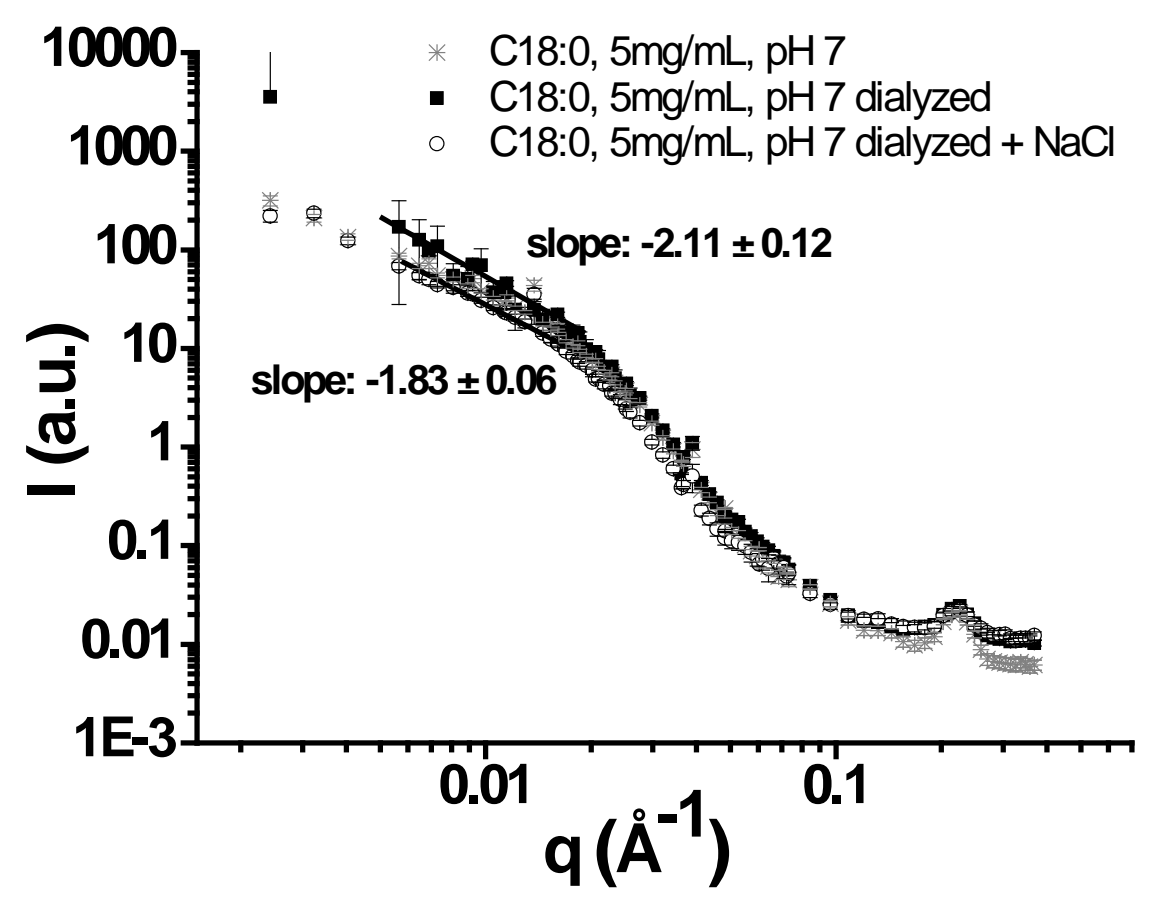

Figure 4 - Log-log representation of the neutron scattered intensity (I(q)) of sophorolipid twisted ribbons before and after dialysis and to a dialyzed sample to which $\mathrm{NaCl}$ has been added.

For q-values larger than about $0.03 \AA^{-1}$ the I(q) spectra becomes very similar. In particular, the diffraction peak at $0.24 \AA^{-1}(\mathrm{~d}=2.65 \mathrm{~nm})$, which is related to the head-to-tail order of sophorolipids within the ribbon plane, is not affected at all by the presence of $\mathrm{NaCl}$ as its position does not vary after dialysis nor after addition of $\mathrm{NaCl}$ to the dialyzed solution. This evidence suggests that the role of $\mathrm{NaCl}$ is not structural, but it rather adsorbs at the ribbon surface thus promoting aggregation. To prove this hypothesis we have performed a complementary experiment, the goal of which is to demonstrate the spatial distribution of the salt. This was done using solid state NMR and the TRAPDOR pulse sequence, which is very useful to probe close proximities $(<\sim 5-8 \AA)^{26,27}$ between dipolar and quadrupolar nuclei, in this case, ${ }^{1} \mathrm{H}$ (spin 1/2) belonging to sophorolipids and ${ }^{23} \mathrm{Na}$ (spin-3/2) ions. As shown by regular TEM images in Figure S1, drying does not seem to affect the integrity of the ribbon chiral structure, and for this reason we assume that the NMR data recorded on the dried sample can provide a good picture of the salt distribution around the ribbons themselves. 

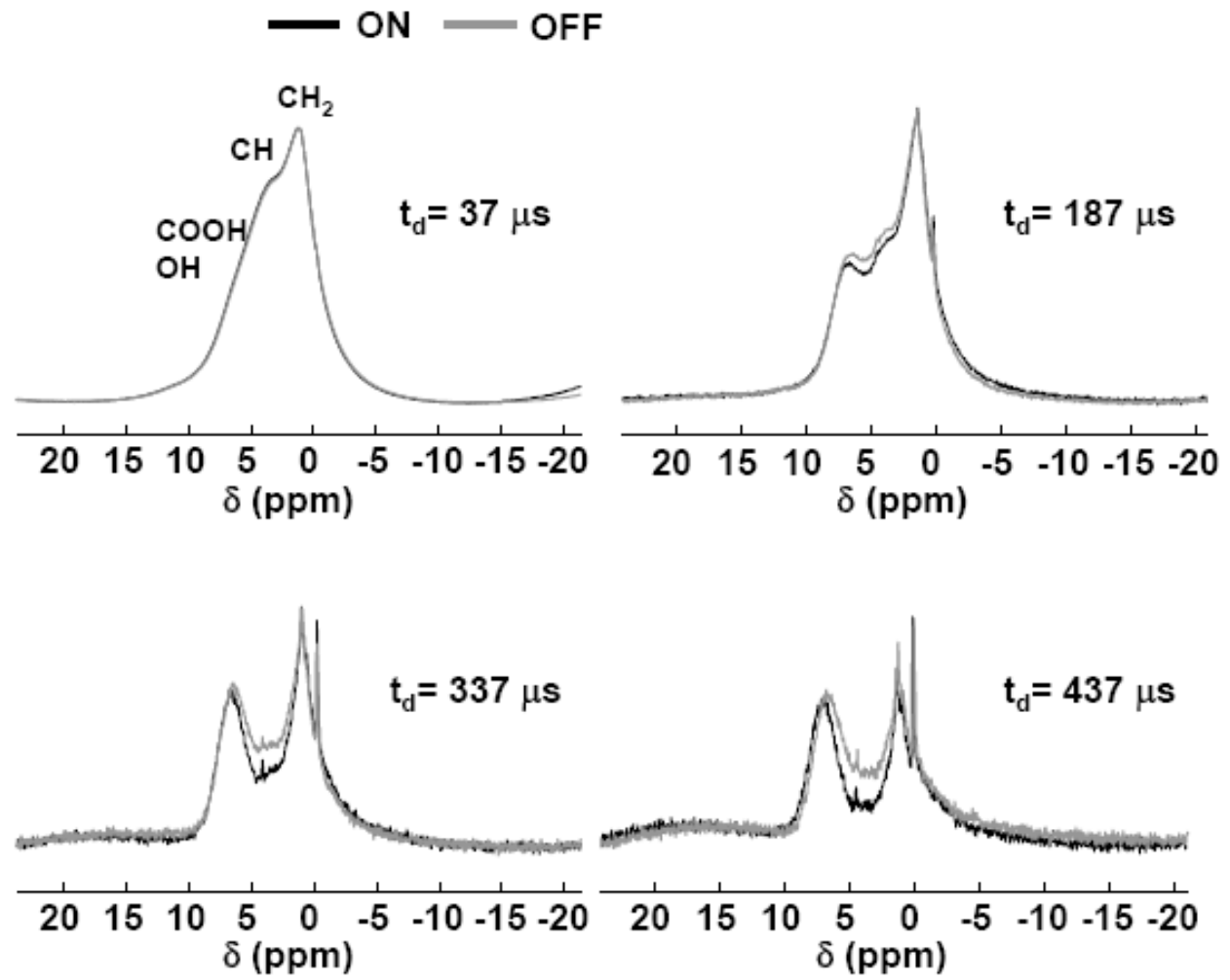

Figure $\left.5-{ }^{1} \mathrm{H}^{23} \mathrm{Na}\right\}$ TRAPDOR solid state NMR experiments performed on sophorolipid twisted ribbon xerogel at four different dephasing times, $t_{d}=37 \mu \mathrm{s}, 187 \mu \mathrm{s}, 337 \mu \mathrm{s}$ and $437 \mu \mathrm{s}$. ON and OFF respectively refer to the irradiation power of the ${ }^{23} \mathrm{Na}$ channel: $\mathrm{OFF}=10^{-12} \mathrm{~W}$ ( $\equiv$ no irradiation), $\mathrm{ON}=138 \mathrm{~W}$ (irradiation).

In Figure 5, we report the typical TRAPDOR experiments recorded on the as-synthesized sophorolipid ribbons xerogel. The ${ }^{1} \mathrm{H}$ NMR signal of the powder is very broad, as expected for a material in the solid state and previously discussed for these materials; ${ }^{14}$ one can nevertheless recognize the signal of the $\mathrm{CH}_{2}$ groups in the aliphatic moiety at $1.3 \mathrm{ppm}$, the sophorose $\mathrm{CH}$ signals between $3 \mathrm{ppm}$ and $4 \mathrm{ppm}$ and the $\mathrm{OH}$ groups belonging to $\mathrm{COOH}$ and sophorose above $5 \mathrm{ppm}$. The effect of ${ }^{23} \mathrm{Na}$ irradiation does not affect at all the sophorolipid ${ }^{1} \mathrm{H}$ signal at very short $\mathrm{t}_{\mathrm{d}}$ times $(37 \mu \mathrm{s})$ as one can see from the matching of the grey and black spectra. However, at longer $t_{d}(187 \mu \mathrm{s})$, the spectrum corresponding to the ${ }^{23} \mathrm{Na}$ irradiation presents a loss on the intensity in the 3-4 ppm region, related to the $\mathrm{CH}$ groups in sophorose. This is very clear by repeating the experiment at various $t_{d}$ times: $337 \mu \mathrm{s}, 437 \mu \mathrm{s}$, showing the $\mathrm{CH}$ groups being the ones that are most affected by the ${ }^{23} \mathrm{Na}$ irradiation. This seems to suggest that upon drying, the sodium ions preferentially locate in the proximity of sophorose. As an additional comment, one should note a loss in the NMR signal at long $t_{d}$ due to short spin-spin relaxation times. In the end, TRAPDOR experiments show that sodium is very close to the sophorolipid ribbon and, upon dry- 
ing, it seems that $\mathrm{Na}^{+}$has preferential interactions with the carbohydrate moiety, as one would most likely expect, as it is known that the affinity of $\mathrm{Na}^{+}$for aliphatic chains is poor. ${ }^{31}$

The data presented above seem to indicate that salt does not play a structural role in the formation of sophorolipid twisted ribbons, as these subsist after its removal. However, salt strongly interacts with the ribbon layer and promotes aggregation of individual ribbons into bundles. At the same time, salt also contributes to stabilize ribbons at their extremities and limit their longitudinal growth, as in the as-synthesized samples non-uniform lengths are also largely observed. Many examples in the literature deal with self-assembled fibrillar structure in which counterions play an important role. Lui et al. found that L-histidine ester terminated bolaamphiphiles are complexed by $\mathrm{Cu}^{2+}$ ions because of the metal coordinating properties of the headgroups and thus, such coupling, promotes nanofibers formation with one-molecular thickness. ${ }^{32}$ Aimé et al. synthesized nucleoamphiphiles through ion exchange between acetate ions of vesicles and nucleotides which spontaneously resulted in chiral helices. ${ }^{33}$ Both examples show that the interaction between amphiphiles and their counterion is essential to obtain the formation of self-assembly fibers. If the counterion is missing, no fibers are formed. However, the important role of counterions is commonly found for charged amphiphiles, electrostatically attracted to oppositely charged counterions. In other cases amphiphiles contain counterion-coordinating sites like imidazole groups. ${ }^{32}$ Saturated sophorolipids, however, neither have charged headgroups (at acidic $\mathrm{pH}$ ) nor do they have prominent metal ions coordinating sites; however, both still spontaneously form chiral fibrils, in the presence or in the absence of a salt, thus indicating that the latter does not play a structureforming role. Similar data can be found in the literature, although they are mainly based on proteins and not on small amphiphilic molecules. The influence of the ionic strength on fibrillar aggregates has been studied for example for bovine $\beta$-lactoglobulin, a major whey protein of cow and sheep's milk. De Vries and coworkers ${ }^{34}$ used atomic force microscopy to show that shorter and more curved fibrils are formed at ionic strength of $\mathrm{I}=100 \mathrm{mM}$ while long and straight fibers were obtained at lower ionic strength (I = $13 \mathrm{mM}$ ), in both cases at $\mathrm{pH} 2$ and $\mathrm{T}=80^{\circ} \mathrm{C}$. The thickness of the fibers was not affected. The origin of this effect can be seen in the electrostatic repulsion between individual $\beta$-lactoglobulin molecules (every molecule has about 20 positives charges). At low ionic strength, the elongation process of the fibers is faster than nucleation which yields too longer fibers. However at high ionic strength, the charge between monomers of $\beta$-lactoglobulin is screened and more nucleation points exist. Nucleation is then faster than elongation and shorter fibers are predominant. Lomakin et al. ${ }^{35}$ and Sabaté et al. ${ }^{36}$ have shown that amyloid $\beta$-proteins, associated with the Alzheimer disease, experience the same trend. They reported that the nucleation/elongation process follows a model of amyloid- $\beta$ fibrillogenesis according 
to which fibers are formed within micelles. Above a critical concentration, the peptide self-assemble into micelles and, once they have reached a critical size, fibrils nucleate within these micelles. Fibrils then grow by binding new monomers entities to fibril ends. ${ }^{37}$ Whether this mechanism applies to the formation of sophorolipid ribbons or not will be discussed elsewhere.

\section{CONCLUSIONS}

This work provides the method to produce infinitely long self-assembled twisted ribbons with a monodispersed cross-section of $13.5 \pm 1.5 \mathrm{~nm}$. The ribbons, initially obtained from a bioderived glycolipid in water at neutral $\mathrm{pH}$, present a natural polydispersity between at least $10 \mathrm{~nm}$ and $40 \mathrm{~nm}$. We show here that by eliminating the residual salt in the parent solution by a simple dialysis step against deionized water, it is possible to improve the quality of the fiber dispersion. We use TEM under cryogenic conditions to visualize the ribbons in their native aqueous environment, thus reducing artifacts due to drying, and to calculate the polydispersity. In addition, we use SANS as a more statistically-reliable mean to prove an improved monodispersity. In fact, we observe a $-2.11 \pm 0.12$ slope in the low-q range of the log-log representation of the spectrum when the dialyzed compound is analyzed, while the slope is only $-1.83 \pm 0.12$ for the as-synthesized compound. A -2 slope is attributed to a flat morphology uniformly distributed throughout the sample, while slight variations from the theoretical value indicate a more fractal morphology, a typical sign of aggregation, as actually shown by cryo-TEM. Two negative controls involving time of maturation in salt-containing system and addition of salt in the dialyzed medium confirm that monodispersity can only be obtained in a salt-free environment.

\section{ACKNOWLEDGMENTS}

The research leading to these results has received funding from the European Community's Seventh Framework Programme (FP7/2007-2013) under Grant Agreement n Biosurfing/289219. The French Région Ile de France SESAME program is acknowledged for financial support (700 MHz NMR spectrometer).

\section{REFERENCES}

\footnotetext{
${ }^{1}$ R. Dominguez, K. C. Holmes, Actin Structure and Function, Annu Rev Biophys, 2011, 40, 169-186

2 J. F. Smith, T. P. J. Knowles, C. M. Dobson, C. E. MacPhee, M. E. Welland, Characterization of the nanoscale properties of individual amyloid fibrils, Proc. Nat. Acad. Sci., 2006, 103, 15806-15811
} 
${ }^{3}$ A. Gautieri, S. Vesentini, A. Redaelli, M. J. Buehler, Hierarchical Structure and Nanomechanics of Collagen Microfibrils from the Atomistic Scale Up, Nano Lett. 2011, 11, 757-766

${ }^{4}$ J. D. Hartgerink, E. Beniash, S. I. Stupp, Peptide-amphiphile nanofibers: A versatile scaffold for the preparation of selfassembling materials, Proc. Nat. Acad. Sci., 2002, 99, 5133-5138

${ }^{5}$ M. M. Giraud-Guille, N. Nassif, F. M. Fernandes, Collagen-based Materials for Tissue Repair, from Bio-inspired to Biomimetic, Ch.5 in Materials Design Inspired by Nature : Function Through Inner Architecture, Eds. P. Fratzl, J. W.C. Dunlop, R. Weinkamer, 2013, Royal society of Chemistry

${ }^{6}$ B. F. Lin,K. A. Megley, N. Viswanathan, D. V. Krogstad, L. B. Drews, M. J. Kade, Y. Qian, M. V. Tirrell, pH-responsive branched peptide amphiphile hydrogel designed for applications in regenerative medicine with potential as injectable tissue scaffolds, J. Mater. Chem., 2012, 22, 19447

${ }^{7}$ V. M. Tysseling-Mattiace, V. Sahni, K. L. Niece, D. Birch, C. Czeisler, M. G. Fehlings, S. I. Stupp, J. A. Kessler, SelfAssembling Nanofibers Inhibit Glial Scar Formation and Promote Axon Elongation after Spinal Cord Injury, J. Neurosci., 2008, 28, 3814-3823

${ }^{8}$ K. Sugiyasu, S.-I. Tamaru, M. Takeuchi, D. Berthier, I. Huc, R. Oda, S. Shinkai, Double helical silica fibrils by sol-gel transcription of chiral aggregates of gemini surfactants, Chem. Commun., 2002, 1212-1213

${ }^{9}$ H. Huo, Y. Li, Y. Yuan, S. Lin, B. Li, M. Wang, Y. Yang Chiral Carbonaceous Nanotubes Containing Twisted Carbonaceous Nanoribbons, Prepared by the Carbonization of Chiral Organic Self-Assemblies, Chem. Asian J. 2014, 9, 2866 - 2871

${ }^{10} \mathrm{M}$. Lämmerhofer, Chiral recognition by enantioselective liquid chromatography: Mechanisms and modern chiral stationary phases, J. Chromatogr. A, 2010, 1217, 814-856

${ }^{11}$ T. Shimizu, Self-Assembled Organic Nanotubes: Toward Attoliter Chemistry, J. Polym. Sci. Part A: Polym. Chem. 2008, 46, 2601-2611

${ }^{12}$ J. P. F. Lagerwall, C. Schuetz, M. Salajkova, J. H. Noh, J. H. Park, G. Scalia, L. Bergström formation to multifunctional thin films, NPG Asia Materials, 2014, 6, e80

${ }^{13}$ S. Vignolini, P. J. Rudall, A. V. Rowland, A. Reed, E. Moyroud, R. B. Faden, J. J. Baumberg, B. J. Glover, U. Steiner, Pointillist structural color in Pollia fruit, Proc. Natl. Acad. Sci. USA 2012 109, 15712-15715

${ }^{14}$ A.-S. Cuvier, J. Berton, C. Stevens, G. C. Fadda, F. Babonneau, I. N. A. Van Bogaert, W. Soetaert, G. Pehau-Arnaudet, N. Baccile, pH-triggered formation of nanoribbons from yeast-derived glycolipid biosurfactants, Soft Matter, 2014, 10, 39503959

${ }^{15}$ R. Oda, I. Huc, M. Schmutz, S. J. Candau, F. C. MacKintosh, Tuningbilayer twistusing chiralcounterions, Nature, 1999, 399, 566-569

${ }^{16}$ E. T. Pashuck, S. I. Stupp, Direct Observation of Morphological Tranformation from Twisted Ribbons into Helical Ribbons, J. Am. Chem. Soc. 2010, 132, 8819-8821

${ }^{17}$ L. Ziserman, H.-Y. Lee, S. R. Raghavan, A. Mor, D. Danino, Unraveling the Mechanism of Nanotube Formation by Chiral Self-Assembly of Amphiphiles, J. Am. Chem. Soc. 2011, 133, 2511-2517

${ }^{18}$ V. Castelletto, I. W. Hamley, R. A. Hule, D. Pochan, Helical-Ribbon Formation by a b-Amino Acid Modified Amyloid bPeptide Fragment, Angew. Chem. Int. Ed. 2009, 48, 2317 -2320

${ }^{19}$ D. Develter, S. Fleurackers, Sophorolipids and Rhamnolipids, in Surfactants from Renewable Resources, M. Kjellin, I. Johansson Eds., 2010, John Wiley \& Sons, Ltd

${ }^{20}$ P. Dhasaiyan, A. Banerjee, N. Visaveliya, B. L. V. Prasad, Influence of the Sophorolipid Molecular Geometry on their Self-Assembled Structures, Chem - Asian J, 2013, 8, 369

${ }^{21}$ N. Baccile, F. Babonneau, J. Jestin, G. Pehau-Arnaudet, I. Van Bogaert Unusual, pH-Induced, Self-Assembly Of Sophorolipid Biosurfactants, ACS Nano, 2012, 6, 4763-4776;

${ }^{22}$ P. Dhasaiyan, P. R. Pandey, N. Visaveliya, S. Roy, B. L. V. Prasad, Vesicle Structures from Bolaamphiphilic Biosurfactants: Experimental and Molecular Dynamics Simulation Studies on the Effect of Unsaturation on Sophorolipid SelfAssemblies, Chem. Europ. J., 2014, 20, 6246-6250

${ }^{23}$ A. Brizard, C. Aime, T. Labrot, I. Huc, D. Berthier, F. Artzner, B. Desbat, R. Oda Counterion, Temperature, and Time Modulation of Nanometric Chiral Ribbons from Gemini-Tartrate Amphiphiles, J. Am. Chem. Soc. 2007, 129, 3754-3762 ${ }^{24}$ I. N. A. Van Bogaert, K. Saerens, C. De Muynck, D. Develter, W. Soetaert, E. J. Vandamme, Microbial production and application of sophorolipids, Appl. Microbiol. Biotechnol., 2007, 76, 23-34

${ }^{25}$ Brulet et al., J. Appl. Cryst. 2007, 40, 165-177

${ }^{26}$ C. A. Fyfe, K. T. Mueller, H. Grondey, K. C. Wong-Moon Solid-state double-resonance NMR experiments involving quadrupolar and spin 1/2 nuclei, J. Phys. Chem., 1993, 97, 13484-13495

${ }^{27}$ C. P. Grey, A. J. Vega, Determination of the Quadrupole Coupling Constant of the Invisible Aluminum Spins in Zeolite HY with 'W7Al TRAPDOR NMR, J. Am. Chem. Soc., 1995, 117, 8232-8242 
${ }^{28}$ L. Ziserman, H.-Y. Lee, S. R. Raghavan, A. Mor, D. Danino, Unraveling the Mechanism of Nanotube Formation by Chiral Self-Assembly of Amphiphiles, J. Am. Chem. Soc. 2011, 133, 2511-2517

${ }^{29}$ I. W. Hamley, Form Factor of Helical Ribbons, Macromolecules 2008, 41, 8948

${ }^{30}$ Chapter 1 in Principles of colloid and surface chemistry, $3^{\text {rd }}$ edition, Eds. P. C. Hiemenz, R. Rajagopalan, 1997, Marcel Dekker editor, New York

${ }^{31}$ C. Zhang, P, Carloni, Salt effects on water/hydrophobic liquid interfaces: a molecular dynamics study, J. Phys.: Condens. Matter,2012, 24, 124109

${ }^{32}$ Y. Liu, T. Wang, Z. Li, M. Liu, Copper(II) ion selective and strong acid-tolerable hydrogels formed by an L-histidine ester terminated bolaamphiphile: from single molecular thick nanofibers to single-wall nanotubes Chem. Commun. 2013, 49, 4767-9.

${ }^{33}$ C. Aimé, R. Tamoto, T. Satoh, A. Grelard, E. J. Dufourc, T. Buffeteau, H. Ihara, R. Oda, Nucleotide-Promoted Morphogenesis in Amphiphile Assemblies: Kinetic Control of Micrometric Helix Formation Langmuir 2009, 25, 8489-8496

${ }^{34}$ L. N. Arnaudov, R. De Vries, Strong Impact of Ionic Strength on the Kinetics of Fibrilar Aggregation of Bovine $\beta$ Lactoglobulin Biomacromolecules 2006, 7, 3490-3498.

${ }^{35}$ A Lomakin, D. S. Chung, G. B. Benedek, D. a Kirschner, D. B. Teplow, On the nucleation and growth of amyloid betaprotein fibrils: detection of nuclei and quantitation of rate constants Proc. Natl. Acad. Sci. U. S. A. 1996, 93, 1125-1129

${ }^{36}$ R. Sabaté, J. Estelrich, Evidence of the Existence of Micelles in the Fibrillogenesis of $\beta$-Amyloid Peptide J. Phys. Chem. B 2005, 109, 11027-11032

${ }^{37}$ D. M. Walsh, A. Lomakin, G. B. Benedek, M. M. Condron, D. B. Teplow, Amyloid beta-protein fibrillogenesis. Structure and biological activity of protofibrillar intermediates J. Biol. Chem. 1997, 272, 22364-22372 